\title{
ISO far infrared observations of the high latitude cloud L 1642 ${ }^{\star}$
}

\section{The density and temperature structure}

\author{
K. Lehtinen ${ }^{1}$, D. Russeil ${ }^{1,2}$, M. Juvela ${ }^{1}$, K. Mattila ${ }^{1}$, and D. Lemke ${ }^{3}$ \\ 1 Observatory, Tähtitorninmäki, PO Box 14, University of Helsinki, 00014 Helsinki, Finland \\ e-mail: kimmo.lehtinen@helsinki .fi \\ 2 Laboratoire d'Astrophysique de Marseille, 2 place Le Verrier, 13004 Marseille, France \\ 3 Max-Planck-Institut für Astronomie, Königstuhl 17, 69117 Heidelberg, Germany
}

Received 16 January 2004 / Accepted 28 April 2004

\begin{abstract}
We have performed a large-scale mapping of the high-latitude, moderate extinction dark cloud L 1642 with ISO (Infrared Space Observatory) at $200 \mu \mathrm{m}$ to study the properties of dust, the virial equilibrium condition of the cloud, and their relation to star formation. The cloud consists of three denser regions which are connected by diffuse material. Only one of the regions, the one most massive and with the greatest optical depth, is related to a temperature mimimum. The pre-main sequence binary stars IRAS 04325-1419 and IRAS 04327-1419, probably born within the cloud, are located close to this temperature mimimum coinciding with the column density maximum. The minimum dust temperature is $\sim 13.8 \mathrm{~K}$. The ratio $2 E_{\mathrm{kin}} /\left|E_{\mathrm{pot}}\right|$ for the star-forming region is about 1.2, and thus it is close to being gravitationally bound. However, the other regions are not gravitationally bound. On a $1^{\prime}-10^{\prime}$ spatial scale the radial density distributions, derived from far-infared optical depth maps, are similar between the star-forming and non star-forming regions. The differences that distinguish the star-forming core appear to be its higher density and lower dust temperature.
\end{abstract}

Key words. infrared: ISM - ISM: individual objects: Lynds 1642 - ISM: individual objects: IRAS 04325-1419 ISM: individual objects: IRAS 04327-1419 - ISM: clouds - ISM: dust, extinction

\section{Introduction}

Translucent high latitude molecular clouds have properties intermediate between diffuse high latitude clouds and dark molecular clouds. Only in rare cases do these clouds have any star formation. Thus the study of such clouds may constrain the physical mechanisms responsible for triggering low mass star formation.

Lynds 1642 is a well-known high galactic latitude $(b=$ $-36.7^{\circ}$ ) translucent/dark cloud, also listed as MBM 20 in Magnani et al. (1985). For a review of the cloud see Liljeström (1991). Hearty et al. (2000) determined a distance of $112-160 \mathrm{pc}$ for L 1642 . In this paper we adopt a distance of $140 \mathrm{pc}$. The maximum optical extinction at the centre has been estimated to be $A_{\mathrm{V}} \approx 8$ mag (Lehtinen et al. 2004) based on near-IR color excesses derived from 2MASS data. An extended lower extinction halo is seen around the central core, visible in extinction, optical scattered light and far-IR $100 \mu \mathrm{m}$ IRAS maps.

^ Based on observations with ISO, an ESA project with instruments funded by ESA Member States (especially the PI countries: France, Germany, The Netherlands and the United Kingdom) and with the participation of ISAS and NASA.
Taylor et al. (1982) made HI observations of a region adjacent to and including L 1642. They suggested that L 1642 is a result of hydrodynamic instability within a larger cometary cloud.

The optical and infrared properties of $\mathrm{L} 1642$ have been studied by Laureijs et al. (1987). Using $60 \mu \mathrm{m}$ and $100 \mu \mathrm{m}$ IRAS data they derived for the region of maximum extinction a temperature of $\sim 21 \mathrm{~K}$ for a $\lambda^{-1}$ emissivity law. However, it is now known that at $60 \mu \mathrm{m}$ much of the emission comes from small grains and thus the ratio $I(60 \mu \mathrm{m}) / I(100 \mu \mathrm{m})$ cannot be used to derive a temperature for the dust grains. Laureijs et al. (1987) showed that the radial density distribution $\rho(r)$ follows the $\rho(r) \propto r^{-2}$ profile in the outer parts, and a flatter profile in the inner parts. A similar conclusion was drawn by Liljeström (1991) and Russeil et al. (2003) from CO data. However, all these studies treated the cloud as a single unit, although it consists of several components. Liljeström (1991) studied the dynamical state of the cloud and found that within the region of ${ }^{13} \mathrm{CO}$ emission the cloud is in virial equilibrium.

Despite the modest opacity of the cloud, two lowluminosity young stellar objects have been detected by IRAS in the core of L 1642; IRAS 04325-1419 and IRAS 04327-1419. Both objects are nebulous low-luminosity pre-main sequence 
binary stars (Sandell et al. 1990; Reipurth \& Heathcote 1990). L 1642 is one of the two high-latitude $(|b|>30 \mathrm{deg})$ clouds known to have star formation; the other one is MBM 12 (Luhman 2001 and references therein).

In addition, there are four other IRAS Point Source Catalog objects within the boundaries of our $200 \mu \mathrm{m}$ map; 04336-1412, 04347-1415, 04342-1444 and 04349-1436, which are detected only at $60 \mu \mathrm{m}, 100 \mu \mathrm{m}, 12 \mu \mathrm{m}$ and $100 \mu \mathrm{m}$, respectively. The $100 \mu \mathrm{m}$-only detections are probably artefacts caused by cirrus structures. The $12 \mu \mathrm{m}$-only detection is probably a field star. There is a non-stellar object on the digitized SERC-J survey image at the position of the $60 \mu \mathrm{m}$-only detection, and thus it is probably a galaxy.

The objective of the present paper is to make a detailed study of dust in a translucent high galactic latitude dark cloud. The $200 \mu \mathrm{m}$ ISO observations, combined with $100 \mu \mathrm{m}$ IRAS/ISSA data, will provide accurate estimates of dust temperature, column density and mass. The $200 \mu \mathrm{m}$ map provides an opportunity to trace by their IR emission the "classical large" dust grains which make up most of the dust mass and are responsible for most of the interstellar extinction in the optical. Far-infrared dust emission is optically thin, and less dependent on effects such as chemical evolution which hamper the analysis of molecular emission line data. The dynamic range of far-IR emission is large, enabling us to study both the low density outer regions of the cloud and the high density central regions.

In this paper we describe the basic properties of the cloud, such as dust temperature, mass, and virial equilibrium. In forthcoming articles we shall combine the far-IR data with visual star-count data, near-IR extinction data, mid-IR ISO and IRAS data, and CO emission line data in order to study the properties of different dust populations and their relationship to molecular gas.

\section{Observations and data reduction}

The observations were made with the ISOPHOT instrument aboard the Infrared Space Observatory (ISO) (Kessler et al. 1996) satellite, using the $\mathrm{C} 200$ detector (Lemke et al. 1996). All observations were made using the observing template PHT22 in raster mode. Basic parameters of the observations are given in Table 1. The data analysis was done using PIA ${ }^{1}$ (ISOPHOT Interactive Analysis) V 8.1 (Gabriel et al. 1997). At the first processing level, the detector ramps were corrected for nonlinearity of the detector response, glitches in ramps were removed by using the two-threshold glitch recognition method, and the ramps were fitted with first-order polynomials. At subsequent levels the signals were deglitched, reset interval correction was applied, signals were linearized for the dependence of detector response on illumination, and orbital positiondependent dark currents were subtracted.

\footnotetext{
${ }^{1}$ The ISOPHOT data presented in this paper were reduced using PIA, which is a joint development by the ESA Astrophysics Division and the ISOPHOT Consortium (the ISOPHOT Consortium is led by the Max-Planck-Institute for Astronomy (MPIA), Heidelberg). Contributing ISOPHOT Consortium institutes are DIAS, RAL, AIP, MPIK, and MPIA.
}

Table 1. The parameters of the individual maps. TDT is the Target Dedicated Time number of the observation, PA is the position angle measured from North to East. Observations were made on March 26th 1998.

\begin{tabular}{rrrrrr}
\hline \hline Filter & TDT & Raster steps & $\begin{array}{r}\text { Map size } \\
{\left[{ }^{\prime}\right]}\end{array}$ & $\begin{array}{r}\text { Grid } \\
{\left[{ }^{\prime \prime}\right]}\end{array}$ & $\begin{array}{r}\text { PA } \\
{\left[{ }^{\circ}\right]}\end{array}$ \\
\hline C_200 & 86200660 & $9 \times 9$ & $27 \times 27$ & 180,180 & 0 \\
C_200 & 86200661 & $9 \times 9$ & $27 \times 27$ & 180,180 & 0 \\
C_200 & 86200762 & $9 \times 9$ & $27 \times 27$ & 180,180 & 0 \\
C_200 & 86201063 & $9 \times 9$ & $27 \times 27$ & 180,180 & 0 \\
C_200 & 86201064 & $9 \times 9$ & $27 \times 27$ & 180,180 & 0 \\
C_200 & 86200765 & $9 \times 9$ & $27 \times 27$ & 180,180 & 0 \\
C_200 & 86201166 & $9 \times 9$ & $27 \times 27$ & 180,180 & 0 \\
C_200 & 86201167 & $9 \times 7$ & $27 \times 21$ & 180,180 & 0 \\
\hline
\end{tabular}

There are 8 maps with a size of $27^{\prime} \times 27^{\prime}$ each, which have been calibrated individually by using the FCS (fine calibration source) measurements bracketing the actual measurement. The typical statistical uncertainty of a map pixel is $0.2 \mathrm{MJy} \mathrm{sr}^{-1}$. The calibration differences between individual maps in the final mosaic have been minimized by simultaneously minimizing the surface brightness differences at each border between maps. The applied relative scaling of individual maps is typically $12 \%$.

To derive the dust temperature we combine our ISO data with IRAS ISSA $100 \mu \mathrm{m}$ data. Because the surface brightness of DIRBE data is more accurately calibrated than that of IRAS, we have scaled the IRAS data to the DIRBE calibration with a relation $I(\mathrm{DIRBE})=0.90 \times I(\mathrm{IRAS})+1.34 \mathrm{MJy} \mathrm{sr}^{-1}$ (for the method see Lehtinen et al. 2003a). This relation is derived from a $6^{\circ}$ diameter circular region around L 1642.

The values of the $200 \mu \mathrm{m}$ surface brightness map have been compared with the DIRBE data. There are 11 DIRBE pixels within the $200 \mu \mathrm{m}$ map. The DIRBE surface brightness values at $100 \mu \mathrm{m}, 140 \mu \mathrm{m}$ and $240 \mu \mathrm{m}$ have been fitted with a modified blackbody function with $\lambda^{-2}$ emissivity law to get values at $200 \mu \mathrm{m}$. The $200 \mu \mathrm{m}$ ISO map has been convolved with the DIRBE beam and color corrected using the temperatures derived from fitting the DIRBE values. The relation between ISO- and DIRBE-based intensities at $200 \mu \mathrm{m}$ is shown in Fig. 1. A linear fit forced to go through the origin gives $I(\mathrm{ISO})=0.82 I(\mathrm{DIRBE})$, which is within the uncertainties of each dataset. Therefore, we prefer to keep the FCS based calibration for the $200 \mu \mathrm{m}$ ISO data.

The angular resolution of the $100 \mu \mathrm{m}$ ISSA map near L 1642 has been determined by fitting some point sources with Gaussians, giving a $F W H M$ resolution $\sim 4.5^{\prime}$. When combining the ISO and IRAS maps we thus convolve the ISO data with a Gaussian function having $F W H M=4.5^{\prime}$. The maps shown in this paper all have the original $90^{\prime \prime} \times 90^{\prime \prime}$ pixel size of the ISOPHOT C200 camera. 


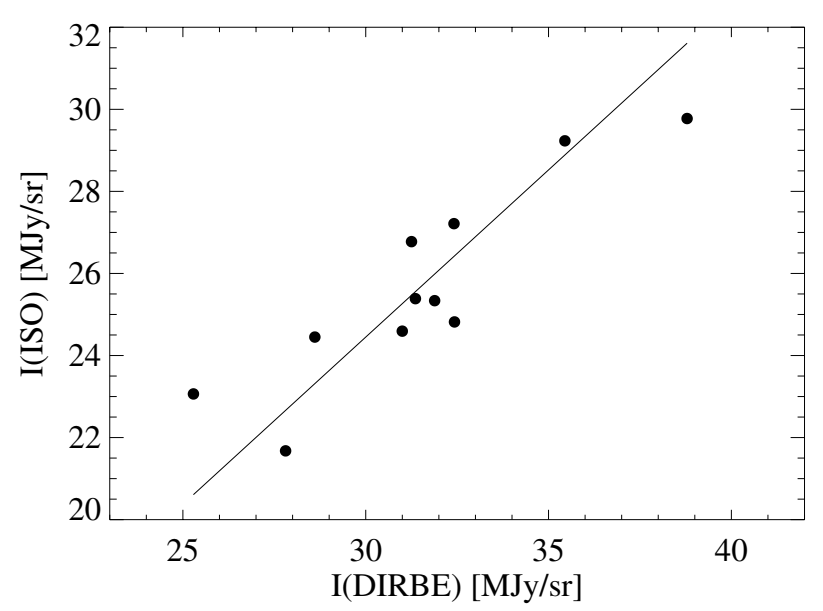

Fig. 1. Relation between $200 \mu \mathrm{m}$ ISO intensity and the $200 \mu \mathrm{m}$ intensity derived from $100 \mu \mathrm{m}, 140 \mu \mathrm{m}$ and $240 \mu \mathrm{m}$ DIRBE intensities (see text). The solid line is a linear fit giving $I($ ISO $)=0.82 I(\mathrm{DIRBE})$.

\section{Results}

\subsection{General structure of $200 \mu \mathrm{m}$ emission}

The $200 \mu \mathrm{m}$ surface brightness map is shown in Fig. 2. The cloud consists of three separate denser regions which are seen in brightness and optical depth maps, and which we have named region $\mathrm{A}, \mathrm{B}$, and $\mathrm{C}$. These regions are interlaced by diffuse dust which extends all the way to the map borders. Region A consists of two maxima separated by 5.3', which we call clump A1 and A2. We have used the $200 \mu \mathrm{m}$ optical depth map to derive the physical dimensions of the regions. Regions B and C have been fitted with two-axial Gaussian surfaces, while the clumps A1 and A2 have been fitted with oneaxial Gaussian surfaces. The positions and FWHM sizes of the fitted Gaussians are given in Table 2, and are plotted in Figs. 2, 3,5 and 6 . The properties of these condensations are discussed in more detail in Sect. 3.4.

There are two filaments of dust extending to the North-East and East from the main cloud. None of the six IRAS sources within our map is detected at $200 \mu \mathrm{m}$, nor any new point source candidates.

\subsection{Temperature distribution}

The temperature of the dust has been derived, pixel by pixel, from the $200 \mu \mathrm{m}$ ISO map and $100 \mu \mathrm{m}$ IRAS ISSA map by using a modified blackbody function

$I(\lambda) \propto \lambda^{-\alpha} B\left(\lambda, T_{\text {dust }}\right)$

where $\alpha$ is the dust emissivity power law index, and $B\left(\lambda, T_{\text {dust }}\right)$ is the Planck function. We assume $\alpha=2$.

The dust temperature map is shown in Fig. 3. The minimum temperature is $\sim 13.8 \mathrm{~K}$. The position of the minimum temperature corresponds well to the position of the optical depth maximum of the region B (see Fig. 5). Region B is the only region in L 1642 which is related to a temperature minimum. The IRAS sources 04325-1419 and 04327-1419 are located close to the temperature minimum.

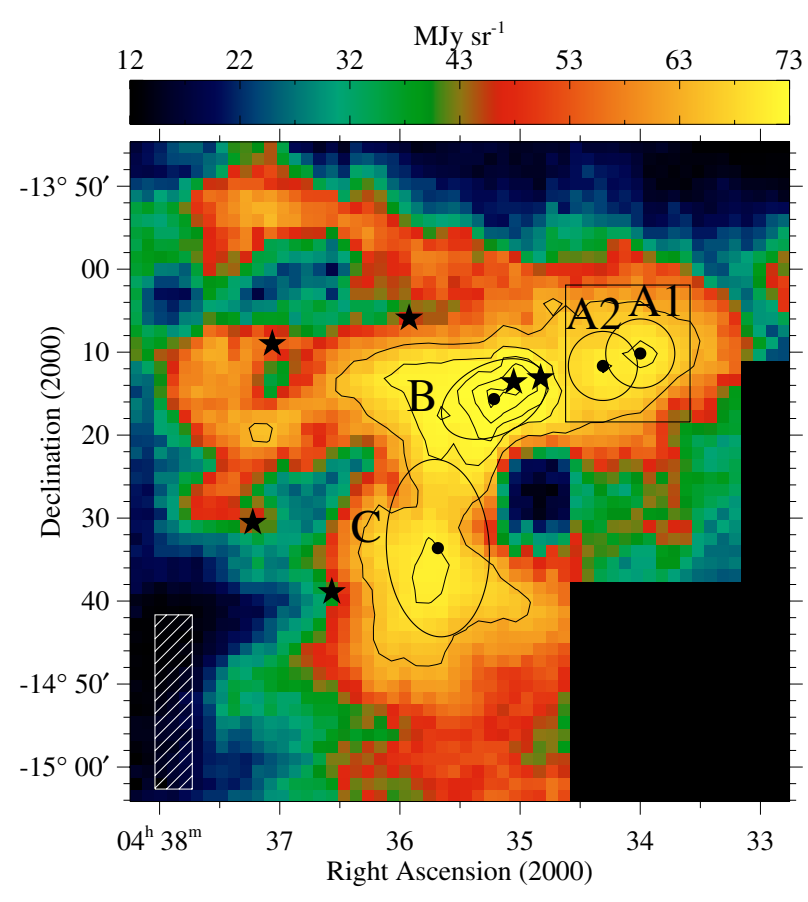

Fig. 2. An image of $200 \mu \mathrm{m}$ surface brightness. The IRAS sources, marked with asterisks, are from right to left: IRAS 04325-1419, 04327-1419, 04336-1412, 04342-1444, 04347-1415 and 04349-1436. The area marked by a rectangle is shown in more detail in Fig. 6. The circles and ellipses show the FWHM sizes of the regions as derived from the $200 \mu \mathrm{m}$ optical depth map. The shaded area shows the region which has been used to determine the background intensity when deriving the temperature and optical depth. The contours are at 30, 40, 50,60 and $70 \mathrm{MJy} \mathrm{sr}^{-1}$. No background intensity has been subtracted in this image. A colour version of the figure is provided in the electronic version of the paper.

The relation between $200 \mu \mathrm{m}$ ISO and $100 \mu \mathrm{m}$ IRAS surface brightness is shown in Fig. 4. The relation diverges into two sections, and shows that regions with brightest $200 \mu \mathrm{m}$ emission are also the coldest. The range of temperatures within the cloud is strictly limited by the outer envelope of the distribution. The solid lines show the surface brightness ratio at the indicated temperatures. Most of the data points with $I(200 \mu \mathrm{m}) \gtrsim 25 \mathrm{MJy} \mathrm{sr}^{-1}$ belong to region $\mathrm{B}$, where the temperature is limited to $\sim 13.8-14.7 \mathrm{~K}$. Elsewhere, the temperature has a maximum value of about $19.0 \mathrm{~K}$.

\subsection{Optical depth distribution}

The optical depth at $200 \mu \mathrm{m}$ has been calculated with the formula

$\tau(200 \mu \mathrm{m})=I(200 \mu \mathrm{m}) / B\left(200 \mu \mathrm{m}, T_{\mathrm{d}}\right)$

which is valid for optically thin emission and where $I$ is the observed intensity, and $B\left(T_{\mathrm{d}}\right)$ is the blackbody intensity at the dust temperature $T_{\mathrm{d}}$. The optical depth map is shown in Fig. 5. The maximum $200 \mu \mathrm{m}$ optical depths of regions $\mathrm{A}, \mathrm{B}$ and $\mathrm{C}$ are about $4 \times 10^{-4}, 2 \times 10^{-3}$ and $4 \times 10^{-4}$, respectively. The IRAS point source closest to the optical depth maximum of region B is IRAS 04327-1419, located about 4' north-west of the maximum. 

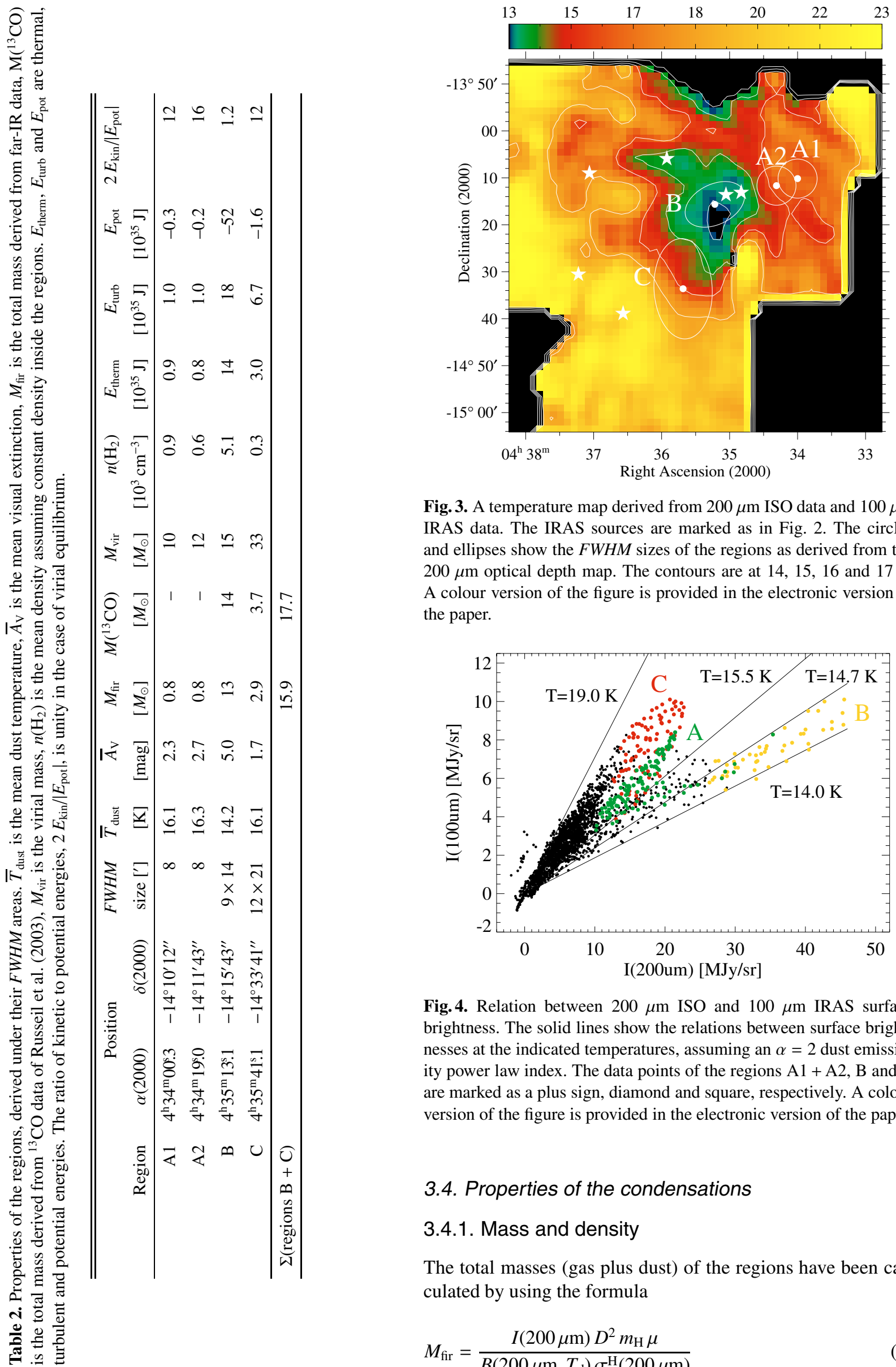

Fig. 3. A temperature map derived from $200 \mu \mathrm{m}$ ISO data and $100 \mu \mathrm{m}$ IRAS data. The IRAS sources are marked as in Fig. 2. The circles and ellipses show the FWHM sizes of the regions as derived from the $200 \mu \mathrm{m}$ optical depth map. The contours are at 14, 15, 16 and $17 \mathrm{~K}$. A colour version of the figure is provided in the electronic version of the paper.

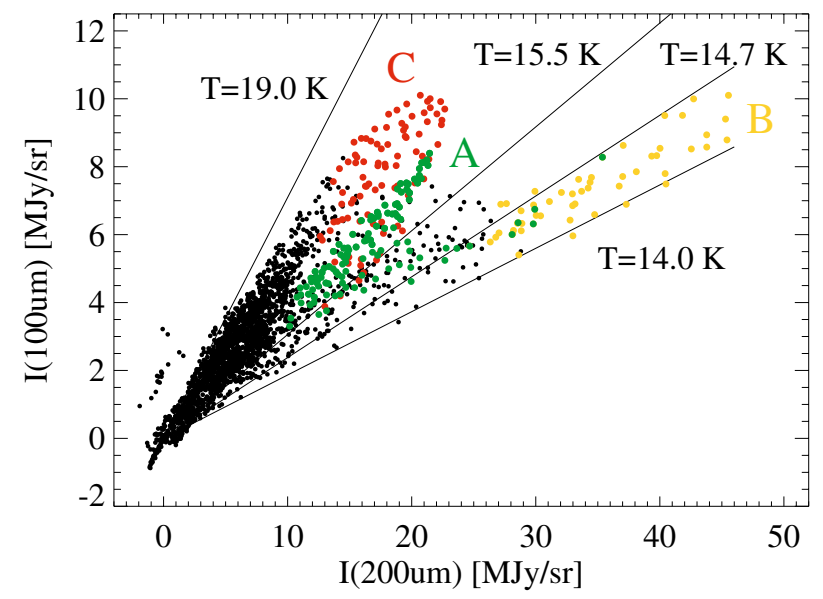

Fig. 4. Relation between $200 \mu \mathrm{m}$ ISO and $100 \mu \mathrm{m}$ IRAS surface brightness. The solid lines show the relations between surface brightnesses at the indicated temperatures, assuming an $\alpha=2$ dust emissivity power law index. The data points of the regions $\mathrm{A} 1+\mathrm{A} 2, \mathrm{~B}$ and $\mathrm{C}$ are marked as a plus sign, diamond and square, respectively. A colour version of the figure is provided in the electronic version of the paper.

\subsection{Properties of the condensations}

\subsubsection{Mass and density}

The total masses (gas plus dust) of the regions have been calculated by using the formula

$M_{\text {fir }}=\frac{I(200 \mu \mathrm{m}) D^{2} m_{\mathrm{H}} \mu}{B\left(200 \mu \mathrm{m}, T_{\mathrm{d}}\right) \sigma^{\mathrm{H}}(200 \mu \mathrm{m})}$ 


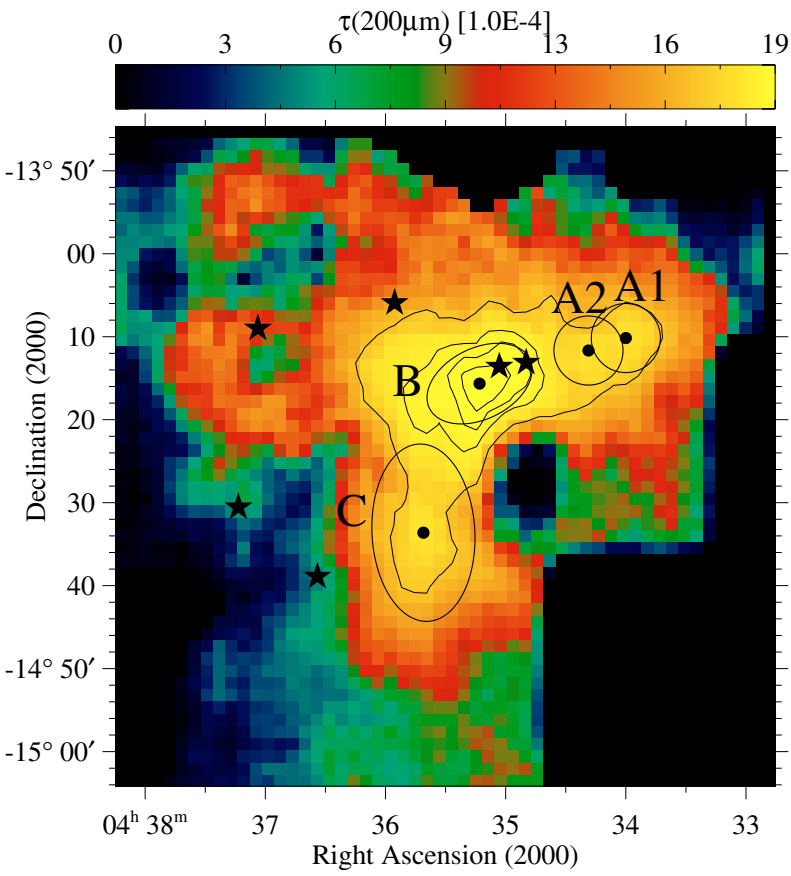

Fig. 5. Optical depth map at $200 \mu \mathrm{m}$. The regions are marked by their fitted $F W H M$ ellipses and circles. The IRAS sources are marked as in Fig. 2. The contours are at 3, 7,11 and $15 \times 10^{-4}$. A colour version of the figure is provided in the electronic version of the paper.

where $I_{200 \mu \mathrm{m}}$ is the observed flux density, $D$ the distance, $m_{\mathrm{H}}$ the mass of a hydrogen atom, $\mu$ the mean molecular weight, $B\left(200 \mu \mathrm{m}, T_{\mathrm{d}}\right)$ the blackbody emission at temperature $T_{\mathrm{d}}$, and $\sigma^{\mathrm{H}}$ the absorption cross section per H-nucleus for which we have used the value $1.5 \times 10^{-25} \mathrm{~cm}^{2}$ (Lehtinen et al. 2004). The masses have been derived by summing the pixels within the FWHM-areas of the regions, and are given in Table 2. We estimate that the total uncertainty of mass, stemming from uncertainties of the factors in Eq. (3) together with uncertainty of the background subtraction, is a factor of two. The total mass of the cloud, summed over the $200 \mu \mathrm{m}$ map after background subtraction, is about $60 M_{\odot}$. This is in excellent agreement with the mass of $59 M_{\odot}$ derived from CO isotopic data by Russeil et al. (2003).

For comparison, we give in Table 2 the total masses of regions $\mathrm{B}$ and $\mathrm{C}$ derived from ${ }^{13} \mathrm{CO}$ data (Russeil et al. 2003). We have assumed for ${ }^{13} \mathrm{CO}$ a fractional abundance of $10^{-6}$ relative to molecular hydrogen. The two mass estimates are in excellent agreement in the case of region $\mathrm{B}$, while for region $\mathrm{C}$ we derive a factor of 1.3 times higher mass from far-IR data. This may imply a lower dust-to-gas ratio, or lower abundance of ${ }^{13} \mathrm{CO}$ in region $\mathrm{C}$ compared to region $\mathrm{B}$, to be discussed in more detail in our forthcoming papers.

Assuming a constant density within the regions, we derive densities of $0.9 \times 10^{3}, 0.6 \times 10^{3}, 5.1 \times 10^{3}$, and $0.3 \times 10^{3} \mathrm{~cm}^{-3}$ for the regions $\mathrm{A} 1, \mathrm{~A} 2, \mathrm{~B}$ and $\mathrm{C}$, respectively.

\subsubsection{Virial equilibrium}

We have studied the virial equilibrium condition of regions B and $\mathrm{C}$ and the clumps $\mathrm{A} 1$ and $\mathrm{A} 2$ by including kinetic and

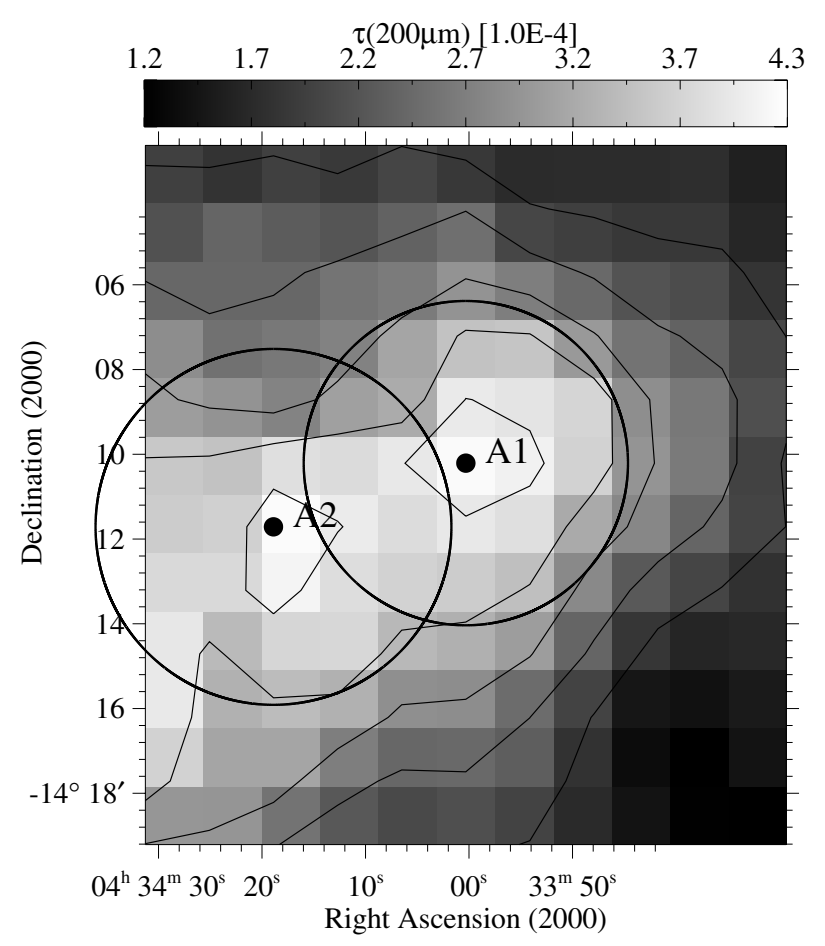

Fig. 6. A combined grayscale and contour image of $200 \mu \mathrm{m}$ optical depth of the region A. The contours are from $2.0 \times 10^{-4}$ to $4.0 \times 10^{-4}$ in steps of $0.5 \times 10^{-4}$. The clumps A1 and A2 are marked, and the circles show their $F W H M$ sizes as derived from Gaussian fitting.

gravitational energies. For the kinetic energy we have included the thermal and the turbulent motions. The thermal energy of molecules is

$E_{\text {therm }}=\frac{3}{2} \mathcal{N} k T$

where $\mathcal{N}$ is the total number of molecules, and $T$ is the kinetic temperature, and $k$ is the Boltzmann constant. The turbulent energy is

$E_{\text {turb }}=\frac{3}{2} M \sigma_{\text {turb }}^{2}$

where $M$ is the cloud mass, and $\sigma_{\text {turb }}$ is the three-dimensional non-thermal (turbulent) velocity dispersion of the molecular gas, $\sigma_{\text {turb }}=\sqrt{\Delta V^{2} /(8 \ln 2)-k T / m}$, where $\Delta V$ is the observed linewidth $(F W H M)$ corrected for opacity line broadening and $m$ is the mass of the molecule under consideration. Because ${ }^{13} \mathrm{CO}$ line includes emission from the halo component around the cloud (Liljeström 1991), it is advisable to derive the velocity dispersion from the $\mathrm{C}^{18} \mathrm{O}$ line, which traces denser gas. However, only region $\mathrm{B}$ is detectable in $\mathrm{C}^{18} \mathrm{O}$ emission, for other regions the velocity dispersion has to be derived from the ${ }^{13} \mathrm{CO}$ line. From the data of Russeil et al. (2003) we derive the following mean values over the $F W H M$-areas of the regions: $\Delta V\left(\mathrm{C}^{18} \mathrm{O}\right)=0.52 \mathrm{~km} \mathrm{~s}^{-1}$ for region $\mathrm{B}, \Delta V\left({ }^{13} \mathrm{CO}\right)=0.67 \mathrm{~km} \mathrm{~s}^{-1}$ for region $\mathrm{C}, \Delta V\left({ }^{13} \mathrm{CO}\right)=$ $0.50 \mathrm{~km} \mathrm{~s}^{-1}$ and $\Delta V\left({ }^{13} \mathrm{CO}\right)=0.53 \mathrm{~km} \mathrm{~s}^{-1}$ for the clumps $\mathrm{A} 1$ and $\mathrm{A} 2$, respectively. For kinetic temperature we have used a value of $10 \mathrm{~K}$ (Russeil et al. 2003). 
The value of potential energy has been derived for a centrally condensed sphere with the formula

$E_{\mathrm{pot}}=-\frac{3}{5} a \frac{G M^{2}}{R}$

where $a=(1-p / 3) /(1-2 p / 5)$ allows for deviation from homogeneous distribution for a spherical cloud which has a radial density distribution of the form $\rho(r) \propto r^{-p}$. We have used $p=1.5$, see Sect. 3.4.3. For the radius $R$ we use the value $R=\sqrt{a b}$, where $a$ and $b$ are the semi-major and semiminor axis of the fitted ellipses, respectively.

The values of thermal, turbulent and potential energies, together with the value of the ratio $2 E_{\mathrm{kin}} /\left|E_{\text {pot }}\right|$ (equal to unity in the case of virial equilibrium), are given in Table 2. For region $\mathrm{B}$ the ratio $2 E_{\text {kin }} /\left|E_{\text {pot }}\right|$ is about 1.2 . Taking into count the relatively large uncertainties related to the values of $D$ and $\sigma^{\mathrm{H}}$, and with the background subtraction in the maps, we can say that region B is close to being gravitationally bound. On the other hand, for region $\mathrm{C}$ and clumps $\mathrm{A} 1$ and $\mathrm{A} 2$ the ratio $2 E_{\mathrm{kin}} /\left|E_{\mathrm{pot}}\right|$ is greater than unity by a factor of much more than two, which indicates that they are not gravitationally bound.

Table 2 includes the virial mass $M_{\text {vir }}$, derived with the formula

$M_{\mathrm{vir}}=\frac{k \sigma^{2} R}{G}$

where $\sigma$ is the three-dimensional velocity dispersion, $R$ is the cloud radius, and $k$ is a factor whose value depends on the radial density distribution (MacLaren 1988). We use a value $k=1.25$ which is midway between the density distributions $\rho(r) \propto r^{-1}$ and $\rho(r) \propto r^{-2}$. The velocity dispersion is given by the formula

$\sigma=\sqrt{3\left(\frac{k T}{\bar{m}}+\left(\frac{\Delta V^{2}}{8 \ln (2)}-\frac{k T}{m}\right)\right)}$

where $\bar{m}$ is the mean molecular mass, and $m$ is the mass of the molecule used for observations, i.e. ${ }^{13} \mathrm{CO}$ or $\mathrm{C}^{18} \mathrm{O}$. For regions $\mathrm{B}$ and $\mathrm{C}$ we derive virial masses of about 15 and $33 M_{\odot}$. Region $\mathrm{B}$ is the only region for which the actual mass is equal to the virial mass within a factor of two, and which thus can be considered to be gravitationally bound. Unless the other regions are bound by magnetic fields and/or external pressure, they are of transient nature.

\subsubsection{Radial density distribution}

Dust emission from L 1642 at $200 \mu \mathrm{m}$ is optically thin, and traces all the dust along a line of sight. The optical depth through the cloud is so low, $\tau_{200 \mu \mathrm{m}} \lesssim 10^{-3}$, that dust at the centre of the cloud cannot be hidden by absorption at the wavelength of our observations. Thus, we can use the optical depth map to derive the radial dust density distribution, in analogy to the method of Yun \& Clemens (1991). This method assumes that the volume density follows a single power law function of the form $\rho(r) \propto r^{-\gamma}$, and that the dust is isothermal. Then, the optical depth $\tau$ as a function of impact parameter $p$ has the form $\tau \propto p^{-\gamma-1}$.

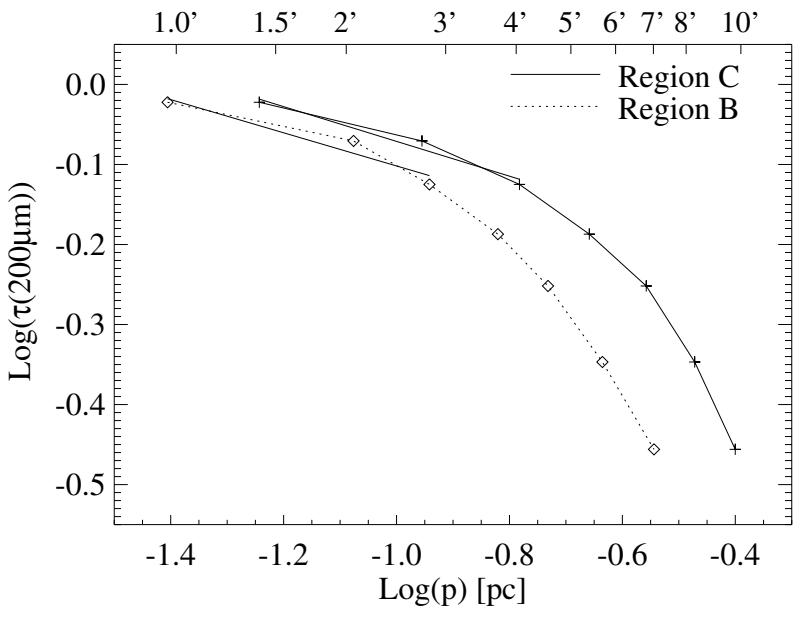

Fig. 7. The observed $200 \mu \mathrm{m}$ optical depth as a function of distance from the center of the region for regions B and C. The optical depths have been normalized to maximum values. The straight lines are fits to the three innermost data points, giving $\alpha \approx 0.2$ (see Sect. 3.4.3).

In order to derive optical depth $\tau$ as a function of the impact parameter $p$ we have used the method where one draws contours on the optical depth map and derives the $\log (\tau(p))=$ $(-\gamma-1) \log (p) \equiv-\alpha \log (p)$ relation from the contours. We have drawn the contours at the 35\%-95\% level of the optical depth maximum, in steps of $10 \%$. The derived radial optical depth profiles of regions B and C, shown in Fig. 7, are similar. In the outer parts the column density profiles steepen, which is partly due to the finite cloud radius (Yun \& Clemens 1991), and partly due to a real change in the value of $\gamma$. Separation of these two effects requires modelling of the cloud, and will be discussed in a forthcoming paper.

In order to derive the value of $\alpha$ we use the three innermost data points, where the effects of finite cloud radius are smallest. For both region $\mathrm{B}$ and region $\mathrm{C}$ we derive the same value, $\alpha=$ 0.2 (see Fig. 7). The derived value of $\alpha$ then corresponds to a radial power law exponent $\gamma_{0}=1+0.2=1.2$ for both regions.

The effect of finite cloud radius on the determination of the value of $\gamma$ is discussed by Yun \& Clemens (1991). The effect is such that the value of $\gamma_{0}$ derived from observations is always an upper limit to the actual value of $\gamma$. The three innermost datapoints sample the cloud up to about 0.25 times the cloud radius. From Fig. 7 of Yun \& Clemens we then estimate a correction factor for $\gamma_{0}$ of about -0.4 . Thus the true value of $\gamma$ is $\gamma \approx 1.2-0.4 \approx 0.8$ for regions $\mathrm{B}$ and $\mathrm{C}$.

\section{Discussion}

High latitude molecular clouds can be divided into three groups depending on their visual extinction; diffuse $\left(A_{\mathrm{V}}<1 \mathrm{mag}\right)$, translucent $\left(1<A_{\mathrm{V}}<5 \mathrm{mag}\right)$ and dark clouds $\left(A_{\mathrm{V}}>5 \mathrm{mag}\right)$ (van Dishoeck \& Black 1988). The maximum visual extinctions of regions $\mathrm{A}, \mathrm{B}$ and $\mathrm{C}$ are about $3 \mathrm{mag}, 8 \mathrm{mag}$ and $2 \mathrm{mag}$, respectively (Lehtinen et al. 2004), when mapping the extinction with a $F W H M=3^{\prime}$ Gaussian beam. Thus region B can be considered as a dark cloud, while the other two regions are translucent. 
In the past, it has been attempted to estimate the equilibrium temperature of "classical large" dust grains by using the IRAS $60 \mu \mathrm{m}$ and $100 \mu \mathrm{m}$ data (Laureijs et al. 1987). The minimum temperature derived by us from $100 \mu \mathrm{m}$ and $200 \mu \mathrm{m}$ data, $\sim 16 \mathrm{~K}$ for a $\lambda^{-1}$ emissivity law, is considerably lower than that derived from $60 \mu \mathrm{m}$ and $100 \mu \mathrm{m}$ data, $\sim 21 \mathrm{~K}$. The difference is caused by the fact that most of the $60 \mu \mathrm{m}$ emission is due to another grain population, the "very small grains", VSGs (Draine \& Li 2001 and references therein).

It is notable that although there is a local column density maximum towards regions $\mathrm{A} 1 / 2$ and $\mathrm{C}$, there is no temperature minimum towards these column density maxima, but instead there is a temperature gradient towards the center of region B, where the global temperature minimum of the cloud is located. We have used the Monte Carlo method of Juvela \& Padoan (2003) to estimate the expected temperature change and to study whether the temperature differences between the regions can be explained solely due to attenuation of the general interstellar radiation field (ISRF). The model consists of a spherical cloud which has a constant density over $10 \%$ of the radius, and a density proportional to $r^{-1.5}$ outside of that. The properties of dust particles are those of the Li \& Draine (2001) model, and the ISRF is the Solar neighbourhood ISRF according to Mezger et al. (1983) and Mathis et al. (1983). A model applied for region A2, with 3 mag of maximum extinction, gives a theoretical temperature drop of about $0.6 \mathrm{~K}$ towards the cloud centre. Such a temperature drop should be discernible in our temperature map. The observed extinction difference between the centers of regions $\mathrm{B}$ and $\mathrm{C}$ is about $6 \mathrm{mag}$, and the observed temperature difference is about $2.6 \mathrm{~K}$. According to radiative transfer calculations a 6 mag difference corresponds to about $1 \mathrm{~K}$ temperature difference. Furthermore, any clumping present in the cloud further reduces the temperature difference. Thus the observed temperature minimum towards region B cannot be explained by extinction only, but a change in the properties of dust particles is required. We conclude that there is a gradual change of dust properties towards the temperature minimum, possibly due to grain coagulation at higher densities.

The derived radial density distributions at the centers of regions $\mathrm{B}$ and $\mathrm{C}$ are not consistent with an infinite isothermal sphere in equilibrium wich has a $\rho \propto r^{-2}$ density distribution, neither with the dynamical inside-out free-fall collapse, which has a $\rho \propto r^{-1.5}$ distribution Shu (1977). Instead, they are more compatible with density distributions of Bonnor-Ebert spheres (Ebert 1955; Bonnor 1956) or of clouds evolved under the influence of ambipolar diffusion (Ciolek \& Mouschovias 1994, 1995; Basu \& Mouschovias 1994, 1995a, 1995b), which have a flatter density distribution at the core.

In general, high latitude molecular clouds are considered to be young and transient. By comparing the observed velocity dispersion of ${ }^{13} \mathrm{CO}$ emission with the expected dispersion for a cloud in virial equilibrium, Liljeström (1991) concluded that $\mathrm{L} 1642$ as a whole is in virial equilibrium. However, the ${ }^{13} \mathrm{CO}$ map of Liljeström covers an area of about $25^{\prime} \times 30^{\prime}$ around core $\mathrm{B}$, but not the regions $\mathrm{A} 1 / 2$ or $\mathrm{C}$. Russeil et al. (2003) have estimated the mass of the cloud based on threedimensional non-LTE modelling of ${ }^{13} \mathrm{CO}$ emission. Although they derived total masses similar to those of Liljeström (1991), they concluded that the mass estimates are uncertain by a factor of about two, due to the unknown density and kinetic temperature distributions. We find that the ratio between virialized and observed mass is about 1.2 for region $\mathrm{B}$, while for other regions it is about 10 . We believe that within uncertainties only region $\mathrm{B}$ can be gravitationally bound, while the other regions of L 1642 are transient.

\subsection{Star formation in L 1642}

Comparison between star forming region B and a non-star forming region $\mathrm{C}$ may elucidate the conditions necessary for onset of low-mass star formation. The regions $\mathrm{B}$ and $\mathrm{C}$ have the following properties:

- both regions are local maxima in the intensity and column density maps;

- the minimum dust temperature of the L 1642 cloud is located at the center of region B. Region C is not associated with a temperature minimum;

- the mean density of region B is over ten times that of region $\mathrm{C}$;

- region B is much closer to virial equilibrium than region C;

- the radial density distributions of region $\mathrm{B}$ and $\mathrm{C}$ are similar.

The fact that the dust temperature decreases towards the cloud center does not mean that the gas kinetic temperature, which determines the thermal energy, would have the same behaviour at these relatively low densities. The $\mathrm{CO}$ isotopic data do not give a definitive clue to the behaviour of the kinetic temperature (Russeil et al. 2003). Thus we cannot estimate the effect of kinetic temperature on star formation in region B.

Based on the above facts, the main reason for star formation in region $\mathrm{B}$ is its much higher volume density, which makes region B gravitationally bound.

\section{Conclusions}

To study the properties of dust in the high latitude cloud Lynds 1642, we have made a wide field $200 \mu \mathrm{m}$ ISOPHOT mapping. By combining our data with $100 \mu \mathrm{m}$ IRAS/ISSA data we have derived maps of dust column density, temperature and mass. We have studied the virial equilibrium condition of the star-forming and non star-forming regions of the cloud. Our main conclusions are:

- The cloud consists of three denser regions, called A1/2, $\mathrm{B}$ and $\mathrm{C}$, and an interclump medium of more tenuous material.

- Only the most massive region $\mathrm{B}$, the one with the highest dust column density, is related to a temperature minimum, $T_{\mathrm{d}} \approx 13.8 \mathrm{~K}$ for a $v^{2}$ emissivity.

- The most massive region is close to being gravitationally bound, while the others are not. Two $\mathrm{T}$ Tauri star binaries are located close to the center of the most massive region, the one that can be classified as a dark cloud. 
- The radial density distributions of the regions B and C, derived from the $200 \mu \mathrm{m}$ optical depth map, are similar on a $1^{\prime}-10^{\prime}$ scale.

- The lower dust temperature in region B cannot be explained solely by the dust attenuation of the general interstellar radiation field, but changes in the properties of dust particles are required.

- The most opaque region B has such a high visual extinction, $\sim 8$ mag, that it can be considered a dark cloud.

Acknowledgements. The work of K.L., M.J. and K.M. has been supported by the Finnish Academy through grants Nos. 17854 and 176071 , which is gratefully acknowledged.

Based on photographic data obtained using The UK Schmidt Telescope. The UK Schmidt Telescope was operated by the Royal Observatory Edinburgh, with funding from the UK Science and Engineering Research Council, until June 1988, and thereafter by the Anglo-Australian Observatory. Original plate material is copyright (C)the Royal Observatory Edinburgh and the Anglo-Australian Observatory. The plates were processed into the present compressed digital form with their permission. The Digitized Sky Survey was produced at the Space Telescope Science Institute under US Government grant NAG W-2166.

We wish to thank the anonymous referee whose comments improved the paper.

\section{References}

Basu, S., \& Mouschovias, T. Ch. 1994, ApJ, 432, 720

Basu, S., \& Mouschovias, T. Ch. 1995a, ApJ, 452, 386

Basu, S., \& Mouschovias, T. Ch. 1995b, ApJ, 453, 271

Bohlin, R. C., Savage, B. D., \& Drake, J. F. 1978, ApJ, 224, 132

Bonnor, W. 1956, MNRAS, 116, 351

Ciolek, G. E., \& Mouschovias, T. Ch. 1994, ApJ, 427, 839

Ciolek, G. E., \& Mouschovias, T. Ch. 1995, ApJ, 454, 194

Draine, B. T., \& Li, A. 2001, ApJ, 551, 807
Ebert, R. 1955, ZA, 37, 217

Gabriel, C., Acosta-Pulido, J., Heinrichsen, I., et al. 1997, in Proc. of the ADASS VI conference, ed. G. Hunt, \& H. E. Payne, ASP Conf. Ser., 125, 108

Hearty, T., Fernández, M., Alcalá, J. M., et al. 2000, A\&A, 357, 681

Juvela, M., \& Padoan, P. 2003, A\&A, 397, 201

Kessler, M. F., Steinz, J. A., \& Anderegg, M. E. 1996, A\&A, 315, L27

Laureijs, R. J., Mattila, K., \& Schnur, G. 1987, A\&A, 184, 269

Lehtinen, K., Lemke, D., Mattila, K., \& Haikala, L. K. 1998, A\&A, 333, 702

Lehtinen, K., Mattila, K., Russeil, D., Lemke, D., \& Haikala, L. K. 2003, Proceedings of the Conference, The Calibration Legacy of the ISO Mission, ed. L. Metcalfe, A. Salama, S. B. Peschke, \& M. F. Kessler, ESA SP-481, 183

Lehtinen, K., et al. 2004, in preparation

Lemke, D., Klaas, U., Abolins, J., et al. 1996, A\&A, 315, L64

Li, A., \& Draine, B. T. 2001, ApJ, 554, 778

Liljeström, T. 1991, A\&A, 244, 483

Lombardi, M., \& Alves, J. 2001, A\&A, 377, 1023

Luhman, K. L. 2001, ApJ, 560, 287

MacLaren, I., Richardson, K. M., \& Wolfendale, A. W. 1988, ApJ, 333,821

Magnani, L., Blitz, L., \& Mundy, L. 1985, ApJ, 295, 402

Mathis, J. S., Mezger, P. G., \& Panagia, N. 1983, A\&A, 128, 212

Mathis, J. S. 1990, ARA\&A, 28, 37

Mezger, P. G., Mathis, J. S., \& Panagia, N. 1982, A\&A, 105, 372

Monet, D. G., Levine, S. E., Canzian, B., et al. 2002, AJ, submitted [arXiv: astro-ph/0210694]

Reach, W., Wall, W. F., \& Odegard, N. 1998, ApJ, 507, 507

Reipurth, B., \& Heathcote, S. 1990, A\&A, 229, 527

Russeil, D., Juvela, M., Lehtinen, K., Mattila, K., \& Paatero, P. 2003, A\&A, 409, 135

Sandell, G., Reipurth, B., \& Gahm, G. 1987, A\&A, 181, 283

Shu, F. H. 1977, ApJ, 214, 488

Taylor, M. I., Taylor, K. N. R., \& Vaile, R. A. 1982, PASA, 4, 440

Yun, J. L., \& Clemens, D. P. 1991, ApJ, 381, 474

van Dishoeck, E. F., \& Black, J. H. 1998, ApJ, 334, 771 